\title{
Topological optimisation of friction dampers for nonlinear resonances mitigation
}

\author{
Enora Denimal ${ }^{1}$, Ludovic Renson $^{1}$, and Loic Salles ${ }^{1}$ \\ Dynamics Group, Imperial College London, London, United Kingdom, \\ enora.denimal@inria.fr, 1.renson@imperial.ac.uk, 1.salles@imperial.ac.uk
}

\begin{abstract}
Friction dampers are commonly used to reduce the vibration amplitude of aircraft turbine blades. However, the shape of such friction dampers can affect significantly damping characteristics and the overall nonlinear dynamic behaviour of the structure. The present work exploits topological optimization to identify damper geometries that minimize response amplitude. The (near-)maximum responses are efficiently computed by solving the harmonic balance equations and a phase quadrature condition. Moving Morphable Components (MMC) are used to describe the damper geometry and an Efficient Global Optimization (EGO) algorithm is used for the optimization.
\end{abstract}

Keywords: topological optimisation, moving morphable component, efficient global optimisation, friction damping

\section{Introduction}

In the design of aircraft turbines, vibration analysis is of major importance to avoid high cycle fatigue failures. Due to the high modal density, resonance cannot be avoided. A classic solution to limit the vibration amplitudes at resonance consists in introducing dry friction damping in the system to dissipate energy. This dissipation can take place in different locations, as shrouds, blades tips or roots, but the most efficient solution is the use of underplatform dampers (UPD) [1]. Located between adjacent blades and maintained in contact due to the centrifugal loading, UPD dissipates energy through friction contact when the blades are vibrating. The UPD geometry at the interface has a significant impact on the damping characteristics and on the overall nonlinear dynamic behaviour of the structure [2]. With the coming of additive manufacturing, new breakthrough UPD geometries could be obtained to improve their efficiency.

Topological optimisation (TO) of continuum structures consists in identifying the part of a given space occupied by material when only the boundary conditions are known [3-5]. It is mostly employed in pre-design stage to identify efficient layouts. The topology of a component is defined by its boundaries between the interior and the exterior, as well as by the number and the location of inner holes. They are optimised simultaneously to minimise an objective function with respect to constraints defined by the user. Two main approaches exist to solve such problems, namely density-based methods and level-set methods. In the 
density-based approach, the density of each element of a mesh are optimised based on their sensitivity to the objective function [3]. In the second approach, the geometry is described implicitly by a Level-Set Function (LSF) propagated by solving an Hamilton-Jacobi equation [3,5]. These two approaches require the sensitivities of the densities or of the shape with respect to the objective function and the constraints. If the sensitivities are analytically unknown, they might be too expensive to determine numerically. In a recent framework called the Moving Morphable Component (MMC), proposed by Guo et al [4], the LSF of the structure is defined as an assembly of several local LSFs. Each of this local LSF is described explicitly through few parameters, which reduces drastically the number of optimisation parameters and makes possible the use of standard optimisation methods such as global optimisation [6].

TO has been used for linear vibrations by optimising the structure eigenvalues [7]. In [8], the cross section of a beam with a geometrical nonlinearity has been optimised to reduce the nonlinear resonant response using a gradientbased approach. Contact problems have been addressed mostly in a quasi-static context [9] using both LSM and density-based approaches. In [10], the MMC framework coupled with kriging-based optimisation was employed to optimise the nonlinear frequency response of blades with an UPD. If promising results were obtained, the computation of the full dynamic response over a large frequency range was required, which resulted in large computational times. Moreover, the contact law was simplistic and did not allow for contact separation, so the variability of the nonlinear dynamic response with regard to the UPD geometry was limited making the optimisation easier.

The present work proposes to reduce the computational cost associated with the nonlinear resonance calculation by directly solving for responses that are in phase quadrature with the applied excitation [11]. A more complex and realistic contact law is considered where contact separation is possible, bringing a softening behaviour and large variations of the nonlinear dynamic response when the UPD geometry varies. Directly tracking the resonance with this approach will allow for exploiting the sensitivity of the resonance and perform robust topological optimisation in the future. Global optimisation is performed and new UPD geometries that divide the level of vibrations by 8 are found at reasonable computational costs.

\section{Mechanical system under study}

The system under study is a $2 \mathrm{D}$ system that simulates the dynamic behaviour of a pair of high-pressure turbine blades [12]. It is displayed in Figure 1(a) and is composed of two blades represented by two beams with platforms. They are connected to a basis that represents the disc. Between the two blades sits the damper which is in contact with the two platforms. In normal working conditions, the latter is kept in this position due to the centrifugal loading. When the blades vibrate, a relative displacement between the platforms and the damper appears. Energy is dissipated by friction, which damps the vibrations. In the rest of the 


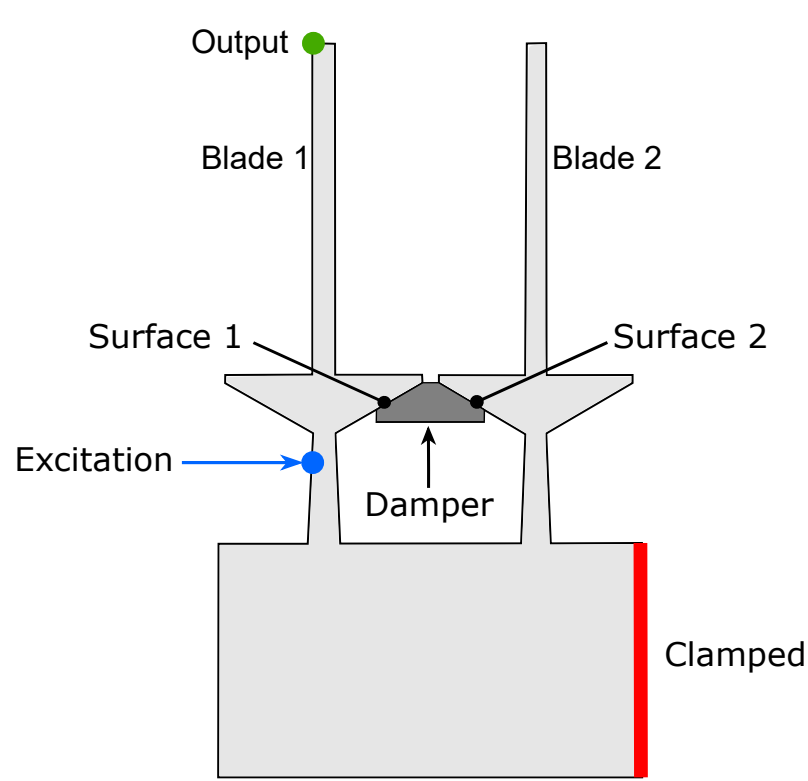

(a) Scheme of the system under study

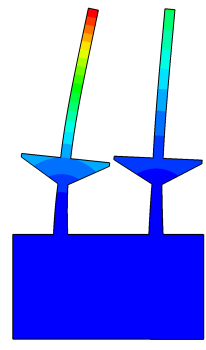

(b) IP mode

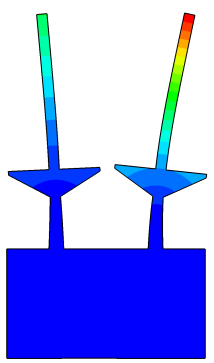

(c) OOP mode

Fig. 1: System under study (a) and the two first bending modes of the blades $(\mathrm{b}, \mathrm{c})$.

study, the system is excited at the basis of blade 1 (see blue point in Figure 1(a)), with an amplitude of $8 \mathrm{~N}$ to activate the contact nonlinearity, and displacements are considered at the blade 1 tip (see green point in Figure 1(a)). The finite element model of the blades is composed of 3324 8-nodes bi-quadratic plane strain elements. The structure is composed of steel with a Young modulus of $197 \mathrm{GPa}$ and a density of $7800 \mathrm{~kg} / \mathrm{m}^{3}$. The first two bending modes of the system correspond to in-phase (IP) and out-of-phase (OOP) motions of the blades and take place at $246.73 \mathrm{~Hz}$ and $247.51 \mathrm{~Hz}$, respectively. They are represented in Figure 1(b,c) respectively. These natural frequencies are determined without the presence of the damper. An initial mesh for the damper is created and is composed of 3604 elements with the same material properties than the blades. The mesh is constructed to ensure matching at the contact points between the platforms and the damper. This mesh will be updated during the topological optimisation as it will be explained in the following.

The two contact surfaces are discretised and a node-to-node contact modelling approach is employed. 2D friction contact elements are employed, one of them consisting of one Jenkins element and one normal spring to allow normal load variations [13]. Each contact element is characterised by four parameters, namely the friction coefficient $\mu$, the tangential contact stiffness $k_{t}$, the normal contact stiffness $k_{n}$ and the normal pre-load $N_{0}$. This contact element allows 
for three different states: stuck, stick/slip and separation. Energy is dissipated by friction during stick/slip. This contact formulation allows the analytical evaluation of the Jacobian matrix. The initial contact pressure is supposed to be homogeneous over the contact surface and depends directly on the centrifugal loading $C_{F}$ and the number of contact points $n_{c o}$ [14]. In the case of a full damper, 51 contact points are present at each contact surface and the normal pre-load is equal to $N_{0}=9.8987 \mathrm{~N}$. The friction coefficient $\mu$ is equal to 0.5 , and the normal contact stiffness is taken equal to $k_{n}=20000 \mathrm{~N} / \mathrm{m}$ for this case. The tangential contact stiffness is chosen equal to the normal contact stiffness.

\section{Geometry description and optimisation process}

\subsection{Geometry parametrisation}

The geometry of the damper is described by a Level-Set Function (LSF) $\Psi$ [3]. This LSF is defined on the design space $\mathcal{D}$ and gives an implicit description of the domain $\Omega$ occupied by the material. $\Psi$ is defined as follows:

$$
\Psi(x, y)=\left\{\begin{array}{l}
\Psi(x, y)>0, \text { if } x \in \Omega \\
\Psi(x, y)=0, \text { if } x \in \delta \Omega \\
\Psi(x, y)<0, \text { if } x \in \mathcal{D} \backslash \Omega
\end{array}\right.
$$

where $(x, y)$ is a point of the design space. Hence, the void is characterised by negative values of the LSF, and the material domain by positive values. The 0 iso-line marks the limit of the material domain. The MMC framework proposes to see this LSF as the union of several "local" LSF $\psi_{i}$ defined explicitly by few parameters [4]. Each local LSF describes an elementary beam that can be moved and deformed. Finally, each local LSF $\psi_{i}$ describes a domain $\Omega_{i}$ occupied by the material and the total domain $\Omega$ occupied by the material is equal to the union of the different subdomains, i.e. $\Omega=\cup_{i} \Omega_{i}$. More concretely, each component $i$ is described by the explicit LSF $\psi_{i}$ given by [4]:

$$
\begin{aligned}
\psi_{i}(x, y)=-\left[\left(\frac{\cos \theta_{i}\left(x-x_{0, i}\right)+\sin \theta_{i}\left(y-y_{0, i}\right)}{L_{i} / 2}\right)^{m}\right. & \\
& \left.+\left(\frac{-\sin \theta_{i}\left(x-x_{0, i}\right)+\cos \theta_{i}\left(y-y_{0, i}\right)}{t_{i} / 2}\right)^{m}-1\right]
\end{aligned}
$$

where $(x, y)$ are the coordinates of a point of the design space, $\theta_{i}$ is the inclination angle of the component, $L_{i}$ its length, $t_{i}$ its thickness and $\left(x_{0, i}, y_{0, i}\right)$ its center, and $m$ is an even number taken equal to 6 here [4]. It describes the sharpness of the LSF. As an example, the geometry of an elementary component is shown Figure 2(a) and its LSF is displayed in Figure 2(b), where negative values are set to zero for better readability. Hence each component is described by a set of five parameters, namely $\theta_{i}, x_{0, i}, y_{0, i}, L_{i}$ and $t_{i}$. By modifying these parameters, one can translate, rotate, shrink or spread the component. And by assembling several components, complex geometries can be described [4] with a very low number 


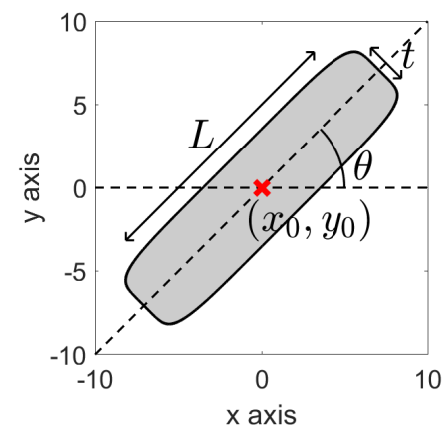

(a)

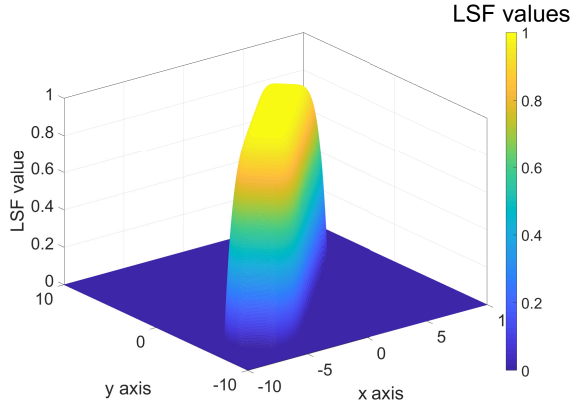

(b)

Fig. 2: (a) A component and its parameters and (b) its Level-Set Function (negative values are set to zero for better readability).

of parameters, hence making the use of gradient-free optimisation methods possible [6].

To get a mechanical translation of the geometry, the global LSF $\Psi$ is then mapped on a mesh. An initial mesh of the damper is created, it is composed of 3604 elements. The LSF is discretised in the center of each element of the damper mesh [5]. Elements that correspond to material (positive value of the LSF) are kept, when elements that correspond to void (negative value of the LSF) are removed to avoid any residual modes in the mode computation. The connectivity of the local LSF is checked and discontinuous geometries are avoided through a penalisation in the optimisation process, explained in the following.

\subsection{Efficient Global Optimisation (EGO) algorithm}

Considering the strong non-convexity of the optimisation problem and the difficulty of the evaluation of the gradients, a global gradient-free optimisation method is adopted, namely the Efficient Global Optimisation (EGO) algorithm [15] which exploits a kriging meta-model of the objective function together with an adaptive sampling process. The general idea is the following: an initial set of inputs (i.e. damper geometries denoted $\mathbf{x}$ ) and outputs (i.e. the amplitude of vibration at resonance peak, denoted $u_{\text {peak }}$, of these geometries) is generated. This set is often called initial learning set. Then, an iterative process is adopted. At each iteration, a kriging meta-model of the objective function is generated first, based on the set of inputs and outputs. Second, this meta-model is exploited to find the point that satisfies a given criterion. The objective function is evaluated for this new point and the learning set is extended with this new point. The iterative process is often stopped when a limit number of iterations is reached. The choice of the criterion to find a new point is important and must balance local and global search to be more precise around possible minima but also to explore unknown parts of the design space. A classic and efficient criterion is the 
Expected Improvement (EI) defined as [15]:

$E[I(\mathbf{x})]=\left(u_{\text {peak }}^{(\min )}-\tilde{u}_{\text {peak }}(\mathbf{x})\right) \Phi\left(\frac{u_{\text {peak }}^{(\mathrm{min})}-\tilde{u}_{\text {peak }}(\mathbf{x})}{s(\mathbf{x})}\right)+s(x) \phi\left(\frac{u_{\text {peak }}^{(\min )}-\tilde{u}_{\text {peak }}(\mathbf{x})}{s(\mathbf{x})}\right)$

where $\mathbf{x}$ is the point of the input space in which the EI is computed, $u_{\text {peak }}^{(\min )}$ is the minimum of the outputs observed so far, $\tilde{u}_{\text {peak }}(\mathbf{x})$ is the kriging prediction at $\mathbf{x}$, $s(\mathbf{x})$ is the prediction standard deviation at $\mathbf{x}, \Phi($.$) is the normal distribution$ function and $\phi($.$) is the normal density function. The new point added in the$ learning set is the point that maximises the EI. Some geometries defined by some parameters might not be connected. To face this issue, in the evaluation of the EI, first the connectivity of the different local LSF $\psi_{i}$ is checked. If the geometry defined by $\mathbf{x}$ is not connected, the EI is penalized with a negative value [6]. This EI maximisation problem is solved with the rgenoud algorithm [16], which couples a genetic algorithm with a gradient evaluation of the EI.

For each new damper geometry, the contact conditions are updated. More particularly, the centrifugal loading directly related to the damper mass is calculated as well as the new contact loading $N_{0}$. The normal contact stiffness $k_{n}$ is also updated so that the ratio $k_{n} / N_{0}$ remains constant over the different geometries [17]. The tangential contact stiffness $k_{t}$ is taken equal to $k_{n}$.

\subsection{Nonlinear analysis}

For each damper geometry, the nonlinear dynamic response is computed. The equation of motion of the problem is given by:

$$
\mathbf{M} \ddot{\mathbf{q}}(t)+\mathbf{C} \dot{\mathbf{q}}(t)+\mathbf{K q}(t)+\mathbf{F}_{n l}(\mathbf{q}(t), \dot{\mathbf{q}}(t))=\mathbf{F}_{e x c}(t)
$$

where $\mathbf{q}$ is the displacements vector, $\mathbf{M}$ is the mass matrix, $\mathbf{C}$ the damping matrix, $\mathbf{K}$ the stiffness matrix, $\mathbf{F}_{n l}$ the vector of the nonlinear forces due to contact and $\mathbf{F}_{\text {exc }}$ the excitation force vector. A Craig Bampton reduction is applied on the damper and on the platform mass and stiffness structural matrices. The number of retained modes is kept constant for numerical convenience and taken high to ensure the quality of the reduction basis on the frequency range of interest. Thus, 12 modes are kept for the platform and 30 for the damper. Contact points, as well as excitation and output points for the platform, are taken as reduction nodes. To take into consideration the structural damping [12], a Rayleigh damping of $0.2 \%$ is added for each component.These different matrices are then assembled to form the global mass, damping and stiffness matrices $\mathbf{M}$, $\mathbf{C}$ and $\mathbf{K}$. It is worth emphasizing here that the size of the matrices is directly related to the damper geometry and to the number of contact nodes. The largest possible size for the system is for the full damper case and is of size 454. During optimisation, the size of the matrices is smaller as less contact points are usually present.

The nonlinear dynamic response of the blades is determined with the Harmonic Balance Method [18]. The displacements are written as a truncated Fourier 
series including $N_{h}$ harmonics. The equation of movement can then be written in the frequency domain and transformed into the problem:

$$
\mathbf{J}_{1}(\mathbf{Q}, \omega)=\mathbf{Z}(\omega) \mathbf{Q}+\tilde{\mathbf{F}}_{n l}(\mathbf{Q})-\tilde{\mathbf{F}}_{e x c}=\mathbf{0}
$$

where $\mathbf{Q}=\left[\mathbf{a}_{0}, \mathbf{a}_{1}, \mathbf{b}_{1}, \ldots, \mathbf{a}_{N_{h}}, \mathbf{b}_{N_{h}}\right]^{T}$ denotes the vector of the cosine and sine coefficient. The resonant solution is directly sought by adding a constraint on the phase $\phi$ between the response and the excitation [11], i.e. the phase must be equal to $\pi / 2$ between the two.:

$$
\mathbf{J}_{2}(\mathbf{Q}, \omega)=\phi-\pi / 2=\mathbf{0}
$$

By adding this constraint, the resonant peak is directly assessed which avoids the computation of the full FRF. The angular frequency also becomes an unknown and the vector of unknown is $\boldsymbol{\alpha}=[\mathbf{Q} ; \omega]$. The problem $\mathbf{J}(\mathbf{Q}, \omega)=$ $\left[\mathbf{J}_{1}(\mathbf{Q}, \omega), \mathbf{J}_{2}(\mathbf{Q}, \omega)\right]=0$ is then solved with a Trust-Region-Dogleg algorithm.

\section{Results}

\subsection{Optimisation parameters presentation}

The number of components describing the damper geometry is chosen equal to five here. To reduce the size of the optimisation problem, a few assumptions are made. First, a component is set to be horizontal and thin at the top of the damper, to ensure that the damper seals the platforms. Second, the damper is assumed to be symmetric about its vertical central axis, which divides by two the number of optimisation parameters. To ensure the existence of the contact between the damper and the platforms, the center of one component must be on the contact line (i.e. the vertical and horizontal coordinates of one component are linked). Finally, with these choices, the optimisation problem is of dimension 9.

The optimisation problem is a minimisation problem, where the objective function is the vibration amplitude at resonance, determined according to Section 3.3. This approach might seem unrealistic as there is no constraint on the damper volume. However, putting a constraint could lead to missing some interesting features. For this reason, no volume constraint are added here.

To ensure a good convergence and a better efficiency of the optimisation, the objective function is slightly modified. Instead of minimising the vibration amplitude at resonance, denoted $u_{\text {peak }}$, the opposite of the inverse of the vibration amplitude at resonance is minimised (i.e. $f_{o b j}=-1 / u_{p e a k}$ ). This choice is motivated by the fact that the vibration amplitude are low as well as their variation. This makes the optimisation difficult as all the points are "compacted". By using the transformation proposed, the points are spread and a small improvement in vibration amplitude corresponds to large variation of the objective function.

\subsection{Optimisation results}

Results are illustrated for one optimisation case. Similar results were obtained for other cases and are not presented here for the sake of concision. The initial 


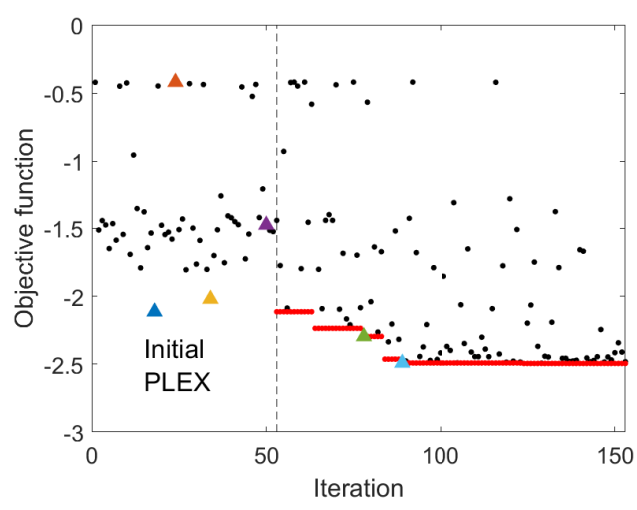

(a) Evolution objective function -

(•) current minimum

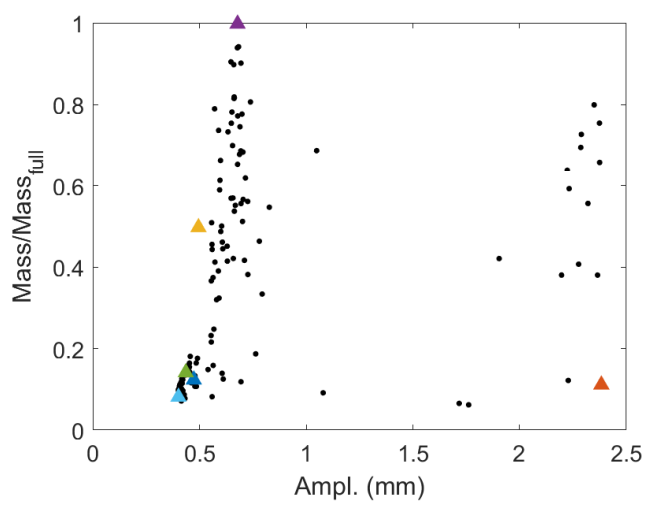

(c) Mass ratio versus vibration amplitude

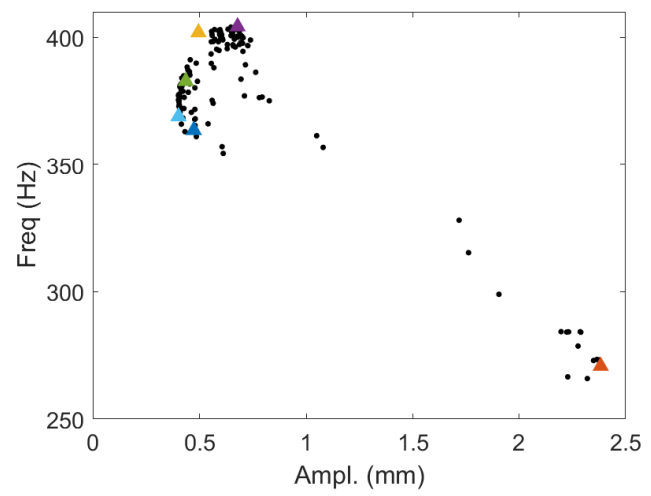

(b) Resonance frequency versus vibration amplitude

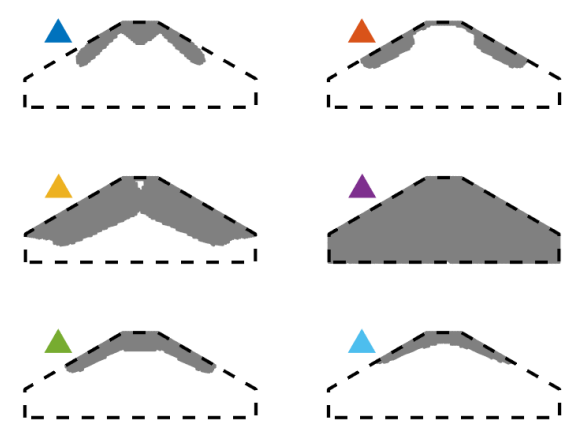

(d) UPD geometries

Fig. 3: Evolution of the objective function (a) - Resonance frequency (b) and mass ratio (c) of the tested configurations - UPD geometries (d) of some cases.

learning set is generated based on a Latin Hypercube Sampling of 200 points of the input space. The non-connected geometries are removed, so it remains 53 initial points. The optimisation loop is stopped after 100 iterations.

Results are shown in Figure 3 where the evolution of the objective function is displayed versus the iteration number (see Figure 3(a)). The current minimum in the optimisation is displayed with red points. The initial learning set is composed of 53 points and the minimum observed in this set is equal to $-2.1 \mathrm{~mm}^{-1}$ (blue triangle), it corresponds to a vibration amplitude equal to $0.47 \mathrm{~mm}$. The corresponding damper geometry is given in Figure 3(d), which corresponds to a very light damper. After that, in 100 iterations, the algorithm has identified new more efficient geometries and the final minimum is equal to $-2.49 \mathrm{~mm}^{-1}$ (light blue triangle). It corresponds to a vibration amplitude equal to $0.4 \mathrm{~mm}$, i.e. the 
vibrations at resonance have been divided by about $15 \%$. The best identified geometry is the light blue one where a large amount of material has been removed. An intermediary geometry is also given (see the green one), the material distribution is somewhat between the bleu and the light blue geometries. This demonstrates the good ability of the approach to identify efficient UPD geometries, but also its capacity to optimise with precision this geometry. This example also shows how sensitive is the dynamic behaviour to the damper geometry.

Another interest of this approach is that many configurations are tested and a map is obtained. It is then possible to observe the properties of other geometries. For example, the resonance frequency and the mass ratio (mass of the considered UPD over the mass of the full damper) are given in Figure 3(b,c). For example, a geometry with a mass reduction of about 50\% with good damping properties is given in yellow. Compared to the other geometries, the total contact surface is present here. As an illustration, the damper geometry associated to the largest vibration amplitude is also given in orange. Its shape is very similar to the optimized one, but a large difference in the dynamic response is observed. As a reference, the full damper case is also illustrated in purple. One can notice that the different geometries presented here (the blue geometries, the yellow and the green ones) have better damping properties (up to $42 \%$ of vibration reduction), and are also lighter (up to $90 \%$ mass reduction). The frequency shift between these geometries is about $50 \mathrm{~Hz}$. Finally, one can identify different geometries with different features and make conception choices based on criterion such as mass reduction, vibrations reduction or frequency shift.

\section{Conclusion and perspectives}

Through this example, we illustrated the good capacity of the MMC approach coupled with EGO algorithm to optimise UPD geometry precisely at reasonable computational costs even with a complex contact law. Because many geometries are tested during the optimisation, it also gives tools for the analysis of the impact of the UPD geometry on the nonlinear dynamic behaviour of the blades. The approach has shown promising results on a simple academic case for global optimisation and more realistic optimisation problems will be considered in the future, by considering a volume constraint for example. The low numerical cost associated to the method and its formulation could be exploited to optimise other nonlinear dynamic features as bifurcation points [19] or to expand the optimisation to robust optimisation.

\section{Acknowledgements}

E. Denimal and L. Salles have received funding from Rolls-Royce and the EPSRC under the Prosperity Partnership Grant CornerStone (EP/R004951/1). L. Renson has received funding from the Royal Academy of Engineering (RF1516/15/11). Rolls-Royce, the EPSRC and the Royal Academy of Engineering are gratefully acknowledged. 


\section{References}

1. Krack M., Salles L., Thouverez, F. Vibration prediction of bladed disks coupled by friction joints. Archives of Computational Methods in Engineering, 24(3), 589-636 (2017).

2. Panning, L., Sextro, W., Popp, K. Optimization of inter-blade friction damper design. Turbo Expo: Power for Land, Sea, and Air, 78576 (2000).

3. Sigmund, O., Maute, K. Topology optimization approaches. Structural and Multidisciplinary Optimization, 48(6), 1031-1055 (2013).

4. Guo X., Zhang W., Zhong W. Doing topology optimization explicitly and geometrically - a new moving morphable components based framework. J. of Applied Mechanics, 81 (2014).

5. V. J. Challis. A discrete level-set topology optimization code written in Matlab. Structural and multidisciplinary optimization, 41(3), 453-464 (2010).

6. Raponi E., Bujny M., Olhofer M., Aulig N., Boria S., Duddeck F. Kriging-assisted topology optimization of crash structures. Computer Methods in Applied Mechanics and Engineering, 348, 730-752 (2019).

7. Díaaz, A.R., Kikuchi, N. Solutions to shape and topology eigenvalue optimization problems using a homogenization method. International Journal for Numerical Methods in Engineering, 35(7), 1487-1502 (1992).

8. Dou, S., Jensen, J. S. Optimization of nonlinear structural resonance using the incremental harmonic balance method. J. of Sound and Vibration, 334, 239-254 (2015).

9. Myśliński, A. Topology optimization of quasistatic contact problems. International Journal of Applied Mathematics and Computer Science 22(2), 269-280 (2012).

10. Denimal E., El Haddad F., Wong C., Salles L. Topological optimisation of underplatform dampers with moving morphable components and global optimisation algorithm for nonlinear frequency response. J. of Engineering for Gas Turbines and Power, In Press (2020).

11. Renson, L., Hill, T. L, Ehrhardt, D. A., Barton, D.A.W., Neild, S.A. Force appropriation of nonlinear structures. Proc. R. Soc. A.474:20170880 (2018).

12. Pesaresi L., Salles L., Jones A., Green J.S., Schwingshackl C.W. Modelling the nonlinear behaviour of an underplatform damper test rig for turbine applications. Mechanical Systems and Signal Processing, 85, 662-679 (2017).

13. Guillen J., Pierre C. An Efficient, Hybrid, Frequency-Time Domain Method for The Dynamics of Large-Scale Dry-Friction Damped Structural Systems. IUTAM symposium on unilateral multibody contacts, 169-178 (1999).

14. Petrov E. P., Ewins D.J. Advanced modeling of underplatform friction dampers for analysis of bladed disk vibration. J. of Turbomachinery, 129(1), 143-150 (2007).

15. Jones D.R., Schonlau M., Welch W.J. Efficient Global Optimization of Expensive Black-Box Functions. J. of Global Optimization, 13, 455-492 (1998).

16. Mebane W., Sekhon J. Genetic Optimization Using Derivatives: The rgenoud package for R. J. of Statistical Software, 42(11), 1-26 (2011).

17. Sextro W. Dynamical contact problems with friction: models, methods and applications. Springer (2002).

18. Detroux T., Renson L., Masset L., Kerschen G. The harmonic balance method for bifurcation analysis of large-scale nonlinear mechanical systems. Computer Methods in Applied Mechanics and Engineering, 296, 18-38 (2015).

19. Alcorta, R., Baguet, S., Prabel, B., Piteau, P., Jacquet-Richardet, G. Period doubling bifurcation analysis and isolated sub-harmonic resonances in an oscillator with asymmetric clearances. Nonlinear Dynamics, 98(4), 2939-2960 (2019). 